\title{
3 Research Square \\ PTEN Loss Correlates with T cell Exclusion across Human Cancers
}

\section{Ziying Lin ( $\nabla$ lzying@mail2.sysu.edu.cn )}

First affiliated hospital of Sun Yat-sen University https://orcid.org/0000-0002-9789-8528

\section{Lixia Huang}

Sun Yat-sen University First Affiliated Hospital

\section{ShaoLi Li}

Sun Yat-sen University First Affiliated Hospital

Jincui Gu

Sun Yat-sen University First Affiliated Hospital

\section{Xiaoxian Cui}

Sun Yet-sen University 8th affiliated hospital

\section{Yanbin Zhou}

Sun Yat-sen University First Affiliated Hospital

\section{Research article}

Keywords: PTEN, PI3K signaling, tumor immune microenvironment, T cells, immunotherapy

Posted Date: May 18th, 2021

DOl: https://doi.org/10.21203/rs.3.rs-531066/v1

License: (c) (i) This work is licensed under a Creative Commons Attribution 4.0 International License. Read Full License

Version of Record: A version of this preprint was published at BMC Cancer on April 19th, 2021. See the published version at https://doi.org/10.1186/s12885-021-08114-x. 


\section{Abstract}

Background: Recent evidences had shown that loss in phosphatase and tensin homolog deleted on chromosome 10 (PTEN) was associated with immunotherapy resistance, which may be attributed to the non-T-cell-inflamed tumor microenvironment. The impact of PTEN loss on tumor microenvironment, especially regarding $T$ cell infiltration across tumor types is not well understood.

Methods: Utilizing The Cancer Genome Atlas (TCGA) and publicly available dataset of immunotherapy, we explored the correlation of PTEN expressing level or genomic loss with tumor immune microenvironment and response to immunotherapy. We further investigated the involvement of PI3K-AKTmTOR pathway activation, which is known to be the subsequent effect of PTEN loss, in the immune microenvironment modulation.

Results: We reveal that PTEN mRNA expression is significantly positively correlated with CD4/CD8A gene expression and $T$ cells infiltration especially $T$ helpers cells, central memory $T$ cell and effector memory $T$ cells in multiples tumor types. Genomic loss of PTEN is associated with reduced CD8+ T cells, type $1 \mathrm{~T}$ helper cells, and increased type $2 \mathrm{~T}$ helper cells, immunosuppressed genes (e.g. VEGFA) expression. Furthermore, $\mathrm{T}$ cell exclusive phenotype is also observed in tumor with $\mathrm{PI} 3 \mathrm{~K}$ pathway activation or genomic gain in PIK3CA or PIK3CB. PTEN loss and PI3K pathway activation correlate with immunosuppressive microenvironment, especially in terms of T cell exclusion. PTEN loss predict poor therapeutic response and worse survival outcome in patients receiving immunotherapy.

Conclusion: These data brings insight into the role of PTEN loss in T cell exclusion and immunotherapy resistance, and inspires further research on immune modulating strategy to augment immunotherapy.

\section{Background}

Phosphatase and tensin homolog deleted on chromosome 10 (PTEN) is well known as the tumor suppressor gene for its negative regulation on phosphatidylinositol 3-kinase (PI3K) pathway[1]. Activated PI3K can convert phosphatidylinositol $(4,5)$ biphosphate (PIP2) into phosphatidylinositol $(3,4,5)$ triphosphate (PIP3), which subsequently activates AKT/mTOR pathway and drives tumor proliferation and progression[2]. PTEN exerts its tumor suppressive function by dephosphorylating PIP3 and thus prevent the activation of PI3K-AKT-mTOR pathway[3]. Other than the negative regulation of oncogene pathway, emerging evidences suggested that PTEN may also play a role in immune modulation and interfering with the therapeutic efficacy of Immunotherapy[4-9].

Immunotherapy that modulate anti-tumor immune response by blocking immune checkpoint is the landmark victory in the treatment of advanced cancer, as accumulating evidences have proven the durable response in multiple tumor types[10]. Marked improvement in clinical outcome had been achieved by checkpoint blockers targeting programmed death-1/programmed death ligand-1 (PD-1/PDL1) in a wide spectrum of cancer entities including non-small cell lung cancer (NSCLC), melanoma etc $[11,12]$. Yet despite the promising clinical benefit and the broad activity, the remaining downside for 
immunotherapy is that only a restricted percentage of patients response to the therapy[13]. That is, intrinsic resistance is widely exist among patients, though the mechanism of which is not clearly understood. Previous studies tried to approach this issue from the perspective of tumor microenvironment (TME) and found that non-T-cell-inflamed tumor microenvironment was closely associate with poor therapeutic response to immunotherapy, particularly with anti-PD-1 antibodies[14, 15]. Great effort is still ongoing to decipher the underlying genomic or molecular mechanism for immune exclusion [16-18].

Among the newly proposed molecular alteration that disfavor T-cell infiltration and contribute to immune evasion, PTEN loss has gain more and more attention as multiple studies have suggested its involvement in immunotherapy resistance. Weiyi Peng etc. first proved that loss of PTEN was associated with reduced number and impaired function of tumor-infiltrating $T$ cells, and poor response to anti-PD- 1 treatment in melanoma patients[9]. Subsequent studies also reported similar finding for other tumor types including NSCLC, triple-negative breast cancer, prostate cancer, glioblastoma, Uterine Leiomyosarcoma etc.[4-8]. With these well-recognized phenomena, emerging studies tried to tackle the specific mechanism of $\mathrm{T}$ cell exclusion mediated by PTEN, and found that PTEN loss was associated with increased expression of certain immune suppressive genes like forkhead box P3 (FOXP3), ID01, VEGFA etc [19]. But still, the downstream molecular pathway by which PTEN mediate tumor immune microenvironment remains a mystery. There is evidence suggesting that PI3K pathway activation after PTEN loss may contribute to T cell exclusion, as PI3K targeting drugs can synergize with immunotherapy in the treatment of melanoma with PTEN loss $[9,20]$. Whether PTEN loss in tumor cause immune exclusion by way of PI3K-AKT-mTOR pathway is yet to be confirmed by further solid evidence.

To investigate the influence of PTEN loss on immune microenvironment across solid tumors, and to decide the involvement of PI3K-AKT-mTOR pathway amidst, we performed an integrative analysis of The Cancer Genome Atlas (TCGA) to clarify the correlation of immune cells infiltration with PTEN loss as well as PI3K-AKT-mTOR pathway activation. We found that both PTEN loss and activation of PI3K pathway were associated with reduced T cell infiltration and enhanced immune suppressive status in multiple tumor types. The correlation of PTEN loss with poor response to immunotherapy was also verified with publicly available data from immunotherapy trials. Our findings bring insight into the novel immunotherapy resistance mechanism associated with PTEN loss, and convey implication for improving immunotherapy efficacy.

Methods

TCGA cancer database

RNA-seq data of 21 solid tumor types from The Cancer Genome Atlas (TCGA) were obtained from GEO database (GEO: GSE62944), where raw data of all the tumor types were reprocessed by aligning the fastq files downloaded from the Cancer Genomics Hub so that the expression value of the genes could be compared between the different samples[21]. Mutation data and copy number variants of PTEN, PIK3CA and PIK3CB were obtained from cBioportal database (https://www.cbioportal.org/). The GISTIC2.0[22] 
annotation of CNAs had been previously binned into $-2,-1,0,1$, and 2, representing total copy loss, hemizygous deletion, euploidy, copy number gain, and high fold amplification. PTEN with loss-of-function mutation or with copy number <-1 were defined as PTEN loss, otherwise defined as PTEN intact. PIK3CA with gain-of-function mutation or with copy number $>1$ were defined as PIK3CA gain, otherwise defined as wide-type PIK3CA. The same rules were also applied for PIK3CB to categorize PIK3CB gain and widetype PIK3CB. Level 3 reverse phase protein array (RPPA) antibody-level protein abundance data (release date January 28, 2016; patch July 14, 2016) produced by The University of Texas MD Anderson Cancer Center were downloaded from TCPA database (https://tcpaportal.org/tcpa/my_protein.html), which mainly consists of TCGA tumor tissue sample sets. Phosphorylation level of AKT, mTOR, STAT3 estimated using median-centered normalized values corresponding to antibody Phospho-Akt (Ser473), Phospho-Akt (Thr308), Phospho-mTOR (Ser2448), Phospho-STAT3 (Tyr705) were obtained from the database. Only part of the included cases have TCPA data available. All the basic information of 21 solid tumors included in the current study were summarized in supplementary Table 1.

Estimation of immune cell enrichment from RNA-seq data

$x$ Cell is a webtool that performs cell type enrichment analysis for different immune and stroma cell types based on the bulk-tissue RNA-seq data. The enrichment abundance of 24 immune cell types in tumor microenvironment was estimated by xCell using Bindea signatures (https://xcell.ucsf.edu/). All TCGA tumors were processed by $x$ Cell to generate enrichment scores for each immune cell type across all samples by integrating single-sample gene set enrichment analysis (sSGSEA) and deconvolution methods[23].

\section{Pathway analysis}

Differential expression of all the genes between tumors with intact PTEN and tumors with PTEN loss in each cancer types were evaluated using DESeq2 package (72) in R software environment. Genes were first ranked according to log2(fold change) and then applied to GSEA analysis using GSEAPreranked tool (GSEA 4.0.3) with a T-cell inflamed signature derived from published literature[24]. Enrichment score of certain pathway activation e.g. PI3K/AKT pathway etc. were computed for each individual tumor based on the normalized transcriptome data of Human gene sets for PI3K/AKT pathway (Reactome_PIP3_activates_AKT_signaling) were obtain from in Molecular Signatures Database v7.0 (MsigDB7.0). Enrichment score was generated by single-sample gene set enrichment analysis (ssGSEA) using R-package (GSEABase and GSVA).

Data obtaining from immunotherapy clinical trial

In order to verify the correlation between PTEN loss and clinical response to immunotherapy, published data of immunotherapy clinical trials (Chen et al., Cancer Discovery 2016[25] and Riaz et al., Cell 2017[26]) with publicly available clinical and transcriptome data were obtained and reanalyzed in the present study. In the Chen et al. cohort, patients with metastatic melanoma were initially treated with CTLA4 blockade and were then treated with PD-1 blockade if they did not respond or progressed on 
CTLA4 blockade. Transcriptome expression of PTEN, CD4, CD8A, VEGFA and clinical data of Chen et al. cohort were retrieved from the supplementary materials of the published study. In the Riaz et al. cohort, a total of 65 patients with advanced melanoma who haven't received any immunotherapy (ipilimumabnaïve) or had progressed on ipilimumab (ipilimumab-progressed) were recruited and treated with PD-1 blockade (Nivolumab). As for Riaz et al. cohort, we only analyzed the transcriptome data derived from specimen biopsied prior to anti-PD-1 treatment. Transcriptome data of Riaz et al. cohort were downloaded from GEO database (GSE91061), while clinical information were obtained from the supplementary material of the corresponding published study. Detailed information of this two datasets were shown in supplementary table $2-3$.

\section{Statistical analysis}

The flow scheme of the whole analysis process was demonstrated in Fig. 1. Assessments of difference in continuous variables between two groups were decided by two-sample t test. Pearson correlation analysis was carried out to determine the correlation between two continuous variables. Logarithmic transformation was carried out for single gene expression to obtain variables that comply normal distribution and suitable for $t$ test or correlation analysis. T test comparison was omitted for certain tumor types when the observed sample size was too small in one group. The prognostic significance of categorical variables was estimated using Kaplan-Meier plots (log-rank test). All statistical analyses and data presentations were performed in R language 3.4.1 (http://www. r-project.org) .

\section{Results}

PTEN expression is positively correlated with T cell infiltration

To investigate the impact of PTEN expression on T cell infiltration, the correlation between mRNA expression of PTEN and T cell specific genes (CD4, CD8A) in different solid tumor were determined by Pearson correlation analysis. As demonstrated in Fig. 2, significant positive correlation between gene expression of PTEN and CD4 was observed in most of the tumor types except for low-grade glioma (LGG), sarcoma (SARC) and thyroid carcinoma (THCA). Similar relationship was also observed between gene expression of PTEN and CD8A, though the correlation was less significant as that between PTEN and CD4.

To further evaluate the impact of PTEN expression on the infiltration of different subpopulations of tumor-infiltrated leukocytes (TILs), we obtain the infiltrating abundance of 24 immune cells, including 13 adaptive immune cells and 11 innate immune cells. The correlation between PTEN mRNA expression and different immune cells infiltrating abundance was determined by Pearson correlation analysis, the yielded results were shown in a dot plot, where the node size indicates the significance of correlation significance and the color denotes the degree of correlation. As shown in Fig. 3, the mRNA expression of PTEN was positive correlated with $T$ helper cells, central memory $T$ cells and effector memory $T$ cells in most of the solid tumors. Specifically, PTEN expression was positively correlated with type 1 T helper (Th1) cells but 
not type $2 \mathrm{~T}$ helper (Th2) cells. It's worth mentioned that no significant positive correlation was observed between PTEN expression and CD8 T cells or cytotoxic cell for most tumor types in the correlation analysis.

PTEN loss is associated with immunosuppressive microenvironment in Pan-cancer

Except for expression level, we want to see whether genomic alteration of PTEN also has an impact on the tumor immune microenvironment. We first classified each tumor sample as PTEN loss or PTEN intact based on it genomic alteration status (as detailly described in the method). Expectedly, PTEN loss was associated with significant lower PTEN expression (supplementary Fig. 1). We compare the infiltrating abundance of immune cells between tumor with PTEN loss or intact PTEN, and found that PTEN loss was associated with significantly reduced infiltration of CD8 T cell, Th1 cell, and increased infiltration of Th2 cell in multiple tumor types (Fig. 4). To further confirm the relevance of PTEN status in T cell inflamed macroenvironment, GSEA pathway analysis was performed to compared the difference of T cell inflamed pathway between tumors with PTEN loss and tumors with intact PTEN. We found that T cell inflamed signature was highly enriched in tumors with wide-type PTEN in most cancer types (supplementary Fig. 2). As for the innate population, dendritic cells (DC), which function as the major antigen presenting cells during $T$ cell activation, was also significantly reduced in multiple tumors with PTEN loss (supplementary Fig. 3A).We also evaluated the association between PTEN loss and the expression of immune suppressive markers that had been previously reported to be associated with PTEN. We did not observed ubiquitous changes in the expression of PDCD1 and CD274 for most tumor types with PTEN loss, with PTEN-loss tumors had elevated PDCD1 and CD274 for some cancer types, but demonstrated downregulated expression in some others and manifested no difference in most cancer types (Fig. 3). Similar findings were also observed for other immune suppressive genes like ID01, FOXP3 and CCL2, CSF1, IL6, etc.(supplementary Fig. 4). However, VEGFA expression was significantly upregulated in tumors with PTEN loss for most of the tumor types, with only a few others demonstrating no significant difference (Fig. 4).

PI3K-AKT-mTOR pathway might contribute to T cell exclusion associated with PTEN loss

It's well known that PTEN loss is associated with activation of PI3K-AKT-mTOR pathway, which had also been linked with immunosuppressive microenvironment and resistance to immunotherapy[9, 27]. Here we further explored the impact of PI3K-AKT-mTOR pathway on tumor immune microenvironment and whether it's the downstream mechanism by which PTEN mediates immune changes in tumor. The activation of PI3K-AKT-mTOR pathway was quantified by ssGSEA score of PI3K pathway geneset (REACTOME_PIP3_ACTIVATES_AKT_SIGNALING), expression level of phosphorylated protein (pAKT[pT308], p-AKT[pS473] and p-mTOR[pS2448]) respectively. We first determined the correlation between PI3K-AKT-mTOR pathway activation and PTEN loss, and found that PTEN loss was associated with significantly increased p-AKT (pT308, pS473) in multiple tumor types, but no changes in p-mTOR (pS2448) for most tumor types. Unexpectedly, PI3K pathway scoring was even reduced in tumors with PTEN loss for multiple tumor types (supplementary Fig. 1). The correlation between PI3K-AKT-mTOR 
pathway activation and immune cell infiltration as well as PTEN expression was determined by Pearson correlation analysis. As shown in Fig. 5, phosphorylation level of AKT(pT308 and pS473) was negatively associated with PTEN expression and T cell infiltration only in several tumor types. No specific correlation with T cell infiltration or PTEN expression was observed for p-mTOR in most tumors. PI3K pathway scoring was negatively associated T cell infiltration but positively correlation with PTEN expression at the same time. PI3K pathway scoring was also positively correlated with memory T cell infiltration, which can owe to its positive correlation with PTEN expression level.

As expression level of p-AKT, p-mTOR or PI3K pathway scoring were not derived from pure tumor mass, but also included those of the infiltrating immune population and other stromal cells. We further focus on the genomic alteration of PI3K, which were more exclusively occur to malignant cells. PIK3CA and PIK3CB are the oncogenic genes that encode the subunits of PI3K, P110a and P110 $\beta$ respectively, the genomic alteration (gain-of-function mutation or copy number amplification) of which had been associated with the development and progression of multiple malignant disease[28, 29]. To further verified the involvement of $\mathrm{PI} 3 \mathrm{~K}$ pathway activation in tumor immune modulation, association between $\mathrm{T}$ cells infiltration and genomic alteration of PIK3CA or PIK3CB was evaluated. Intriguingly, genomic gain in PIK3CA or PIK3CB were associated decreased CD8 T cells, Th1 cell but increased Th2 cells infiltration in multiple tumor types (Fig. 6), which was similar to what we observed for PTEN loss.

PTEN expression predict response to immunotherapy

Prognostic impact of PTEN in patients underwent surgical treatment was evaluated based on TCGA database, where no survival difference was observed across most tumor types (supplementary Fig. 5). To further verified the significance of PTEN in immunotherapy, the correlation between PTEN expression and response to check point blockade was further evaluated in two independent cohort (Chen et al. cohort and Riaz et al. cohort). For patients with advanced melanoma receiving anti-CTLA-4 treatment $(n=18)$ in Chen et al. cohort, cases with low mRNA PTEN expression responded poorly to anti-CTLA4 blockade (Fig. 7A). Similarly, for patients receiving anti-PD-1 treatment in Chen et al. cohort $(n=23)($ Fig. 7A) and Riaz et al. cohort $(n=49)$ (Fig. 7B), non-responders were predominately among those with low mRNA PTEN expression. Of note, tumors with low PTEN expression also demonstrate low CD4/CD8A mRNA expression and high VEGFA mRNA expression at the same time, which is consistent with the findings in TCGA cohort.

We further explored the prognostic significance of PTEN in patients receiving immunotherapy. For patients with advanced melanoma receiving anti-PD- 1 treatment $(n=49)$ in Riaz et al. cohort, overall survival were compared between patients with low PTEN expression (the bottom fifth, $n=10$ ) and high PTEN expression (the top four fifths, $n=39$ ). Patients with low PTEN expression demonstrated worse overall survival $(P=0.1)$ (Fig. $7 C)$.

\section{Discussion}


Defined by lack of T cell infiltration within tumor, non-T-cell-inflamed microenvironment, was one of the major cause for immunotherapy resistance. Our study collected evidence that PTEN loss was one of the tumor-intrinsic mechanism contributing to an immune exclusion phenotype. We profiled the correlation of PTEN loss with T-cell-exclusion microenvironment across different solid tumors based on TCGA database, and found that both genomic loss (loss-of-function mutation or copy number deletion) and low expression of PTEN were associated with immune exclusion in a pan-cancer setting. PTEN's impact on tumor immune microenvironment might be mediated by PI3K pathway, whose activation was also associated immunosuppressive phenotype in multiple solid tumors. These findings not only facilitate our understanding in the correlation between PTEN loss and immunotherapy resistance, but also inspire continued pursuit on immune modulating strategy to augment immunotherapy.

In this study, we evaluated the correlation of genomic alteration and expression of PTEN with tumor T cells infiltration. Consistent with previous findings, we found both genomic loss or reduced expression of PTEN were associated with decreased T cell trafficking and immune suppressive microenvironment in board range of malignancies $[8,9,19,30]$. What differs our findings from the published studies is that we first reported T cell infiltration level was positively correlated with PTEN expression level. Also, reduced expression in PTEN predicted poor response to immunotherapy and worse outcome. These findings indicated that PTEN's impact on immune microenvironment is expression dependent. A recent published study indicated that overexpression of PTEN in tumor cells can enhance T-cell mediated tumor clearance, which also proved that it's the expression level rather than functional status of PTEN that matters[31]. Another interesting finding in the present study is the strong correlation between PTEN expression and memory $T$ cells (central memory $T$ cells and effector memory $T$ cells). Studies on TME had revealed that memory $T$ cells were mainly located in tertiary lymphoid structures (TLS), which encompass abundant immature T cells, B cells and play an important role in tumor cell clearance[32]. The presence of TLS is not only associated with improved clinical outcome in multiple cancers, but also found to promote immunotherapy response in patients with melanoma or renal cell carcinoma[33, 34]. It's possible that the involvement of PTEN loss in immunotherapy resistance is partial mediated by TLS. If it's the case, it can explain the finding reported in a recent study that tumors with wide type PTEN manifested significant higher effector T cells as compared to tumors with mutant PTEN only after the treatment of immunotherapy, when memory $T$ cells will be activated and differentiate into functional T cells [7].

Of note, the analysis of PTEN's correlation with immune infiltration from the perspective of expression level and genomic alteration status didn't yield exactly concordant results. For example, we didn't find significant correlation of PTEN expression with the infiltrating level of CD8 T cell and Th2 cell, which though, were significant reduced and increased respectively in multiple tumors with genomic PTEN loss. Also, memory T infiltration was strongly correlated with PTEN expression, but only demonstrated significant reduction in selected tumor types with PTEN loss. These discrepancy may be attributed to the miscellaneous signal of PTEN that derived from stromal cells in addition to cancer cells. When immune cells or other stromal cell would also contribute to part of the expression level of PTEN, genomic data of PTEN (mutation and copy number variants) were more likely restricted to the tumor cells. Therefore, although we observed a positive correlation between PTEN expression and memory T cells infiltration, we 
can't rule out the possibility that correlation is partially attributed to the high expression of PTEN in certain immature $T$ cells. Another explanation for this discordance could be the way we defined loss-offunction mutation for PTEN. Loss-of-function mutations include mutations that cause inhibition of PTEN catalytic activity or mutations that lead to unstable truncated proteins. As the immune modulating function of PTEN might be independent of its catalytic activity, loss-of-function mutation is not necessary synonymous to loss of immune modulating function[35].

Existing study had shown that PTEN loss in tumor cells were associated with increased expression of immunosuppressive cytokines, especially VEGF, which reduce $T$ trafficking as well as its cytotoxic function in TME [9]. Loss of PTEN in glioma was also reported to be associated with increased PD-L1 expression and immune escape[36]. There is still evidence indicates the correlation between PTEN loss on tumor cells and elevation of immunosuppressive markers like ID01, FOXP3, CCL2, CSF1 ect.[37, 38]. Consistent with the published findings, we also found that PTEN loss is associated with increased mRNA expression of VEGFA, ID01, IL6, CCL2, CSF1 in a wide spectrum of solid tumors. The expression level of PD-1, PD-L1 and FOXP3 were not altered by PTEN loss in most tumors, or even demonstrated reduced expression in some tumors, which might be attributed to the reduced T cells abundance associated with PTEN loss.

Our findings confirmed that PTEN loss in tumor cells was associated with T cell exclusion and an immunosuppressive microenvironment in solid tumors, though the underlying mechanism of which remains a puzzle. As we all know, the cornerstone discovery of PTEN's biology was the negative regulation of the pro-oncogenic PI3K-AKT-mTOR pathway mediated by dephosphorylation of substrate $\mathrm{PIP}_{3}[2,27,39,40]$. Quite a few studies had tried to tackle the immune modulating mechanism of PTEN from the perspective of PI3K-AKT-mTOR pathway alteration. James S. Waldron etc. found that PTEN loss associated with activation of the PI3K-Akt-mTOR pathway and led to a autologous T-cell apoptosis in glioblastoma, which can be diminished by treatment with inhibitors of PI3K-Akt-mTOR pathway[41]. Another study also indicated that PTEN-depleting melanoma cells promoted the expression of immunosuppressive cytokines in a PI3K-dependent manner[37]. We do observed a negative correlation of p-AKT level and PI3K pathway scoring with T cell infiltration in multiple tumor types, though the correlation is not as strong as we expected. It's worth mentioned that the data regarding p-AKT level and PI3K pathway scoring were derived from tumor cells as well as the infiltrating immune cells, which can obscure the correlation between T cell filtration and PI3K pathway activation in tumor cells. What's more, we observed similar changes on $T$ cell infiltration in tumors with genomic gain in PIK3CA/PIK3CB as that in tumors with PTEN loss, which support the hypothesis that PI3K activation and PTEN loss yield similar impact on tumor immune microenvironment. All these findings indicated that the immune modulating effect of PTEN is at least partially attributed to PI3K pathway alteration, although some non-canonical functions of PTEN might also play a role amidst. As indicated in our data, PTEN loss is not synonymous with PI3K-Akt-mTOR activation, which is also revealed in published literature[42]. Except for the typical downregulation of PI3K-Akt-mTOR pathway, PTEN also exerts a series of non-canonical functions in a enzymatic or non-enzymatic manner[27, 35, 43, 44]. Emerging studies revealed that PTEN loss in prostate 
cancer resulted in an immunosuppressive tumor microenvironment through the activation of the Janus kinase 2 (JAK2) - signal transducer and activator of transcription 3 (STAT3) pathway and the subsequent secretion of immunosuppressive chemokines[38]. There are also studies reported that PTEN loss induced secretion of immunosuppressive cytokines via the activation of NF-KB pathway[37, 45]. The exact pathway by with PTEN exerts the immune modulating function is yet to be verified by further studies.

In addition to PTEN loss, more and more oncogenic events had been associated with tumor intrinsic resistance to immunotherapy. WNT/b-catenin pathway activation is also constantly detected in tumor developing resistance to immunotherapy[30,46]. A recent published study has proven the correlation between wnt/b-catenin pathway activation and immune exclusion across human cancers[47]. Also, gainof-function mutations in FGFR3, as well as activation of PPAR-y pathway, have been associated with the T cell exclusion in tumor microenvironment in bladder cancer[18]. EGFR mutation in lung cancer is a wellknown oncogene that impairs response to immunotherapy, which is attributed to an uninflamed phenotype and weak immunotherapy[48-50]. Of course, the above-mentioned oncogenic events didn't account for all the tumors with the non-T-cell-inflamed phenotype, the full picture of which warrants continued effort on further research.

We found that PTEN status was a robust predictor for response to immunotherapy including PD-1 blockade and CTLA-4 blockade, although it was not associated with prognosis among patients without receiving immunotherapy. Our findings demonstrate that PTEN functions at the interface between cancer and tumor microenvironment and can eventually alter therapeutic outcome of immunotherapy. In the era of immunotherapy and precision medicine, our findings will translate into the molecular approach to classify the subpopulation of cancer patients that have greater chance of responding to immunotherapy. Also, with the implication on the mechanism by which PTEN modulate the immune microenvironment, we might be able to develop strategy to manipulate immune landscape and augment the therapeutic efficacy of immunotherapy in the clinical practice. Inhibitors targeting PI3K-Akt-mTOR pathway had shown promising efficacy in improving immunotherapy response in selected cancer type[9, 41, 51]. However, considering the multifaceted function of PTEN in all kinds of biological process, further study is warranted to bring in-depth understanding into its role in immune microenvironment and immunotherapy before we can actually exploit these knowledge in clinical practice.

Even with the promising findings, limitations of the present study need to be clearly addressed. First of all, the current work was merely based on the in silico analysis of TCGA, with all the immune parameters inferred from the transcriptome data. All these findings need to be confirmed by further study integrating IHC data or protein data. Also, data regarding PTEN status or PI3K-Akt-mTOR alteration were not derived from pure tumor cells, but from the tumor mass containing tumor cells as well as a small subset of stromal cells, infiltrating immune cells, whose confounding effect cannot be ruled out. Lastly, our analysis regarding genomic alteration of PTEN is limited to point mutations, small insertions and deletions, as well as copy number variants, whereas other types of genomic changes such as gene fusions or large-scale structural variants remain to be explored. 


\section{Conclusions}

In conclusion, the present study is the first to explore the immunosuppressive effect of PTEN and its potential mechanism in a pan-cancer setting. This set of data sufficiently motivated further effort on unraveling the detailed mechanism of immune exclusion mediated by PTEN loss, and developing immune modulating strategy to augment immunotherapy.

\section{Abbreviations}

BLCA:Bladder Urothelial Carcinoma; BRCA:Breast invasive carcinoma; COAD:Colon adenocarcinoma; GBM:Glioblastoma multiforme; HNSC:Head and Neck squamous cell carcinoma; KICH:Kidney Chromophobe; KIRC:Kidney renal clear cell carcinoma; KIRP:Kidney renal papillary cell carcinoma; LGG:Brain Lower Grade Glioma; LIHC:Liver hepatocellular carcinoma; LUAD:Lung adenocarcinoma; LUSC:Lung squamous cell carcinoma; OV:Ovarian serous cystadenocarcinoma; PAAD:Pancreatic adenocarcinoma; PRAD:Prostate adenocarcinoma; READ:Rectum adenocarcinoma; SARC:Sarcoma; SKCM:Skin Cutaneous Melanoma; STAD:Stomach adenocarcinoma; THCA:Thyroid carcinoma; UCEC:Uterine Corpus Endometrial Carcinoma; Th1:type $1 \mathrm{~T}$ helper cells; Th2:type 2 T helper cells; Tem:effector memory T cells; Tcm:central memory T cells; TFH:follicular T helper cells; TReg:regulatory T cells; Tgd:үठ T cells; NK cells:nature killer cells; DC:dendritic cells; aDC:activated dendritic cell; pDC:plasmacytoid dendritic cell; iDC:immature dendritic cell.

\section{Declarations}

\section{Ethical approval and consent to participate}

The study has been performed in accordance with the ethical standards. This article does not contain any studies with human participants or animals performed by any of the authors. All the data applied in this study are available on pubic dataset that can be accessed without requiring any permission.

\section{Consent for publication}

Not applicable.

\section{Availability of data and materials}

All the data applied in the present study were obtained from the publicly available databases: cBioportal database (https://www.cbioportal.org/); GEO database (GSE62944 https://www.ncbi.nlm.nih.gov/geo/query/acc.cgi?acc=GSE62944); TCPA database (https://tcpaportal.org/tcpa/my_protein.html). 


\section{Competing interests}

The author has no conflict of interests.

\section{Funding}

The present study was supported by grants from the National Natural Science foundation of China (grant no. 81570008).

\section{Author Contributions}

ZL associated with conceptualization, methodology, formal analysis, and original draft preparation. LH and SL participated in validation of analysis and editing of the article. JG and XC participated in data curation. YZ was in charge of supervision, Project administration, funding acquisition, reviewing and Editing of the final manuscript. All authors read and approved the final manuscript.

\section{Author details}

${ }^{1}$ Department of Respiratory and Critical Care Medicine, The First Affiliated Hospital of Sun Yat-sen University, Guangzhou, China; ${ }^{2}$ Department of Respiratory Medicine, The 8th Affiliated Hospital of Sun Yat-sen University, Shenzhen, China.

\section{Acknowledgements}

Not Applicable

\section{References}

1. Lee JO, Yang H, Georgescu MM, Di Cristofano A, Maehama T, Shi Y, Dixon JE, Pandolfi P, Pavletich NP. Crystal structure of the PTEN tumor suppressor: implications for its phosphoinositide phosphatase activity and membrane association. Cell. 1999;99(3):323-34.

2. Manning BD, Toker A. AKT/PKB Signaling: Navigating the Network. Cell. 2017;169(3):381-405.

3. Garcia-Cao I, Song MS, Hobbs RM, Laurent G, Giorgi C, de Boer VC, Anastasiou D, Ito K, Sasaki AT, Rameh L, et al. Systemic elevation of PTEN induces a tumor-suppressive metabolic state. Cell. 2012;149(1):49-62.

4. Guibert N, Jones G, Beeler JF, Plagnol V, Morris C, Mourlanette J, Delaunay M, Keller L, Rouquette I, Favre G, et al. Targeted sequencing of plasma cell-free DNA to predict response to PD1 inhibitors in advanced non-small cell lung cancer. Lung cancer (Amsterdam Netherlands). 2019;137:1-6. 
5. Barroso-Sousa R, Keenan TE, Pernas S, Exman P, Jain E, Garrido-Castro AC, Hughes ME, Bychkovsky $\mathrm{B}$, Umeton R, Files JL, et al: Tumor mutational burden and PTEN alterations as molecular correlates of response to PD-1/L1 blockade in metastatic triple-negative breast cancer. Clinical cancer research: an official journal of the American Association for Cancer Research 2020.

6. Wang C, Feng Y, Zhang C, Cheng D, Wu R, Yang Y, Sargsyan D, Kumar D, Kong AN. PTEN deletion drives aberrations of DNA methylome and transcriptome in different stages of prostate cancer. FASEB journal: official publication of the Federation of American Societies for Experimental Biology. 2020;34(1):1304-18.

7. Zhao J, Chen AX, Gartrell RD, Silverman AM, Aparicio L, Chu T, Bordbar D, Shan D, Samanamud J, Mahajan A, et al. Immune and genomic correlates of response to anti-PD-1 immunotherapy in glioblastoma. Nature medicine. 2019;25(3):462-9.

8. George S, Miao D, Demetri GD, Adeegbe D, Rodig SJ, Shukla S, Lipschitz M, Amin-Mansour A, Raut CP, Carter SL, et al. Loss of PTEN Is Associated with Resistance to Anti-PD-1 Checkpoint Blockade Therapy in Metastatic Uterine Leiomyosarcoma. Immunity. 2017;46(2):197-204.

9. Peng W, Chen JQ, Liu C, Malu S, Creasy C, Tetzlaff MT, Xu C, McKenzie JA, Zhang C, Liang X, et al. Loss of PTEN Promotes Resistance to T Cell-Mediated Immunotherapy. Cancer discovery. 2016;6(2):202-16.

10. Topalian SL, Hodi FS, Brahmer JR, Gettinger SN, Smith DC, McDermott DF, Powderly JD, Carvajal RD, Sosman JA, Atkins MB, et al. Safety, activity, and immune correlates of anti-PD-1 antibody in cancer. N Engl J Med. 2012;366(26):2443-54.

11. Rizvi NA, Peters S. Immunotherapy for Unresectable Stage III Non-Small-Cell Lung Cancer. N Engl J Med. 2017;377(20):1986-8.

12. Eggermont AMM, Blank CU, Mandala M, Long GV, Atkinson V, Dalle S, Haydon A, Lichinitser M, Khattak A, Carlino MS, et al. Adjuvant Pembrolizumab versus Placebo in Resected Stage III Melanoma. N Engl J Med. 2018;378(19):1789-801.

13. Gide TN, Wilmott JS, Scolyer RA, Long GV. Primary and Acquired Resistance to Immune Checkpoint Inhibitors in Metastatic Melanoma. Clinical cancer research: an official journal of the American Association for Cancer Research. 2018;24(6):1260-70.

14. Ji RR, Chasalow SD, Wang L, Hamid O, Schmidt H, Cogswell J, Alaparthy S, Berman D, Jure-Kunkel M, Siemers NO, et al. An immune-active tumor microenvironment favors clinical response to ipilimumab. Cancer immunology immunotherapy: Cll. 2012;61(7):1019-31.

15. Ayers M, Lunceford J, Nebozhyn M, Murphy E, Loboda A, Kaufman DR, Albright A, Cheng JD, Kang SP, Shankaran V, et al. IFN-gamma-related mRNA profile predicts clinical response to PD-1 blockade. J Clin Investig. 2017;127(8):2930-40.

16. Rooney MS, Shukla SA, Wu CJ, Getz G, Hacohen N. Molecular and genetic properties of tumors associated with local immune cytolytic activity. Cell. 2015;160(1-2):48-61.

17. Grasso CS, Giannakis M, Wells DK, Hamada T, Mu XJ, Quist M, Nowak JA, Nishihara R, Qian ZR, Inamura K, et al. Genetic Mechanisms of Immune Evasion in Colorectal Cancer. Cancer discovery. 
2018;8(6):730-49.

18. Sweis RF, Spranger S, Bao R, Paner GP, Stadler WM, Steinberg G, Gajewski TF. Molecular Drivers of the Non-T-cell-Inflamed Tumor Microenvironment in Urothelial Bladder Cancer. Cancer immunology research. 2016;4(7):563-8.

19. Vidotto T, Saggioro FP, Jamaspishvili T, Chesca DL, Picanco de Albuquerque CG, Reis RB, Graham $\mathrm{CH}$, Berman DM, Siemens DR, Squire JA, et al. PTEN-deficient prostate cancer is associated with an immunosuppressive tumor microenvironment mediated by increased expression of ID01 and infiltrating FoxP3 + T regulatory cells. Prostate. 2019;79(9):969-79.

20. Peng W, Williams LJ, Xu C, Melendez B, McKenzie JA, Chen Y, Jackson HL, Voo KS, Mbofung RM, Leahey SE, et al. Anti-OX40 Antibody Directly Enhances The Function of Tumor-Reactive CD8(+) T Cells and Synergizes with PI3Kbeta Inhibition in PTEN Loss Melanoma. Clinical cancer research: an official journal of the American Association for Cancer Research. 2019;25(21):6406-16.

21. Rahman M, Jackson LK, Johnson WE, Li DY, Bild AH, Piccolo SR. Alternative preprocessing of RNASequencing data in The Cancer Genome Atlas leads to improved analysis results. Bioinformatics. 2015;31(22):3666-72.

22. Mermel CH, Schumacher SE, Hill B, Meyerson ML, Beroukhim R, Getz G. GISTIC2.0 facilitates sensitive and confident localization of the targets of focal somatic copy-number alteration in human cancers. Genome biology. 2011;12(4):R41.

23. Aran D, Hu Z, Butte AJ. xCell: digitally portraying the tissue cellular heterogeneity landscape. Genome biology. 2017;18(1):220.

24. Ayers M, Lunceford J, Nebozhyn M, Murphy E, Loboda A, Kaufman DR, Albright A, Cheng JD, Kang SP, Shankaran V, et al. IFN- $\gamma-$ related mRNA profile predicts clinical response to PD-1 blockade. J Clin Investig. 2017;127(8):2930-40.

25. Chen PL, Roh W, Reuben A, Cooper ZA, Spencer CN, Prieto PA, Miller JP, Bassett RL, Gopalakrishnan V, Wani K, et al. Analysis of Immune Signatures in Longitudinal Tumor Samples Yields Insight into Biomarkers of Response and Mechanisms of Resistance to Immune Checkpoint Blockade. Cancer discovery. 2016;6(8):827-37.

26. Riaz N, Havel JJ, Makarov V, Desrichard A, Urba WJ, Sims JS, Hodi FS, Martín-Algarra S, Mandal R, Sharfman WH, et al. Tumor and Microenvironment Evolution during Immunotherapy with Nivolumab. Cell. 2017;171(4):934-49.e916.

27. Salmena L, Carracedo A, Pandolfi PP. Tenets of PTEN tumor suppression. Cell. 2008;133(3):403-14.

28. Jia S, Liu Z, Zhang S, Liu P, Zhang L, Lee SH, Zhang J, Signoretti S, Loda M, Roberts TM, et al. Essential roles of $\mathrm{PI}(3) \mathrm{K}-\mathrm{p} 110$ beta in cell growth, metabolism and tumorigenesis. Nature. 2008;454(7205):776-9.

29. Zhao JJ, Liu Z, Wang L, Shin E, Loda MF, Roberts TM. The oncogenic properties of mutant p110alpha and p110beta phosphatidylinositol 3-kinases in human mammary epithelial cells. Proc Natl Acad Sci USA. 2005;102(51):18443-8. 
30. Trujillo JA, Luke JJ, Zha Y, Segal JP, Ritterhouse LL, Spranger S, Matijevich K, Gajewski TF. Secondary resistance to immunotherapy associated with beta-catenin pathway activation or PTEN loss in metastatic melanoma. J immunother Cancer. 2019;7(1):295.

31. Russell L, Swanner J, Jaime-Ramirez AC, Wang Y, Sprague A, Banasavadi-Siddegowda Y, Yoo JY, Sizemore GM, Kladney R, Zhang J, et al. PTEN expression by an oncolytic herpesvirus directs T-cell mediated tumor clearance. Nature communications. 2018;9(1):5006.

32. Dieu-Nosjean MC, Giraldo NA, Kaplon H, Germain C, Fridman WH, Sautes-Fridman C. Tertiary lymphoid structures, drivers of the anti-tumor responses in human cancers. Immunological reviews. 2016;271(1):260-75.

33. Sautes-Fridman C, Petitprez F, Calderaro J, Fridman WH. Tertiary lymphoid structures in the era of cancer immunotherapy. Nature reviews Cancer. 2019;19(6):307-25.

34. Helmink BA, Reddy SM, Gao J, Zhang S, Basar R, Thakur R, Yizhak K, Sade-Feldman M, Blando J, Han $\mathrm{G}$, et al. B cells and tertiary lymphoid structures promote immunotherapy response. Nature. 2020;577(7791):549-55.

35. Lee YR, Chen M, Pandolfi PP. The functions and regulation of the PTEN tumour suppressor: new modes and prospects. Nature reviews Molecular cell biology. 2018;19(9):547-62.

36. Parsa AT, Waldron JS, Panner A, Crane CA, Parney IF, Barry JJ, Cachola KE, Murray JC, Tihan T, Jensen MC, et al. Loss of tumor suppressor PTEN function increases B7-H1 expression and immunoresistance in glioma. Nature medicine. 2007;13(1):84-8.

37. Ying H, Elpek KG, Vinjamoori A, Zimmerman SM, Chu GC, Yan H, Fletcher-Sananikone E, Zhang H, Liu $\mathrm{Y}, \mathrm{Wang} \mathrm{W}$, et al. PTEN is a major tumor suppressor in pancreatic ductal adenocarcinoma and regulates an NF-kappaB-cytokine network. Cancer discovery. 2011;1(2):158-69.

38. Toso A, Revandkar A, Di Mitri D, Guccini I, Proietti M, Sarti M, Pinton S, Zhang J, Kalathur M, Civenni $G$, et al. Enhancing chemotherapy efficacy in Pten-deficient prostate tumors by activating the senescence-associated antitumor immunity. Cell reports. 2014;9(1):75-89.

39. Keniry M, Parsons R. The role of PTEN signaling perturbations in cancer and in targeted therapy. Oncogene. 2008;27(41):5477-85.

40. Leslie NR, Batty IH, Maccario H, Davidson L, Downes CP. Understanding PTEN regulation: PIP2, polarity and protein stability. Oncogene. 2008;27(41):5464-76.

41. Waldron JS, Yang I, Han S, Tihan T, Sughrue ME, Mills SA, Pieper RO, Parsa AT. Implications for immunotherapy of tumor-mediated T-cell apoptosis associated with loss of the tumor suppressor PTEN in glioblastoma. Journal of clinical neuroscience: official journal of the Neurosurgical Society of Australasia. 2010;17(12):1543-7.

42. Blanco-Aparicio C, Renner O, Leal JF, Carnero A. PTEN, more than the AKT pathway. Carcinogenesis. 2007;28(7):1379-86.

43. Vivanco I, Palaskas N, Tran C, Finn SP, Getz G, Kennedy NJ, Jiao J, Rose J, Xie W, Loda M, et al. Identification of the JNK signaling pathway as a functional target of the tumor suppressor PTEN. Cancer cell. 2007;11(6):555-69. 
44. Song MS, Salmena L, Pandolfi PP. The functions and regulation of the PTEN tumour suppressor. Nature reviews Molecular cell biology. 2012;13(5):283-96.

45. Bezzi M, Seitzer N, Ishikawa T, Reschke M, Chen M, Wang G, Mitchell C, Ng C, Katon J, Lunardi A, et al. Diverse genetic-driven immune landscapes dictate tumor progression through distinct mechanisms. Nature medicine. 2018;24(2):165-75.

46. Spranger S, Bao R, Gajewski TF. Melanoma-intrinsic beta-catenin signalling prevents anti-tumour immunity. Nature. 2015;523(7559):231-5.

47. Luke JJ, Bao R, Sweis RF, Spranger S, Gajewski TF. WNT/beta-catenin Pathway Activation Correlates with Immune Exclusion across Human Cancers. Clinical cancer research: an official journal of the American Association for Cancer Research. 2019;25(10):3074-83.

48. Herbst RS, Baas P, Kim DW, Felip E, Perez-Gracia JL, Han JY, Molina J, Kim JH, Arvis CD, Ahn MJ, et al. Pembrolizumab versus docetaxel for previously treated, PD-L1-positive, advanced non-small-cell lung cancer (KEYNOTE-010): a randomised controlled trial. Lancet. 2016;387(10027):1540-50.

49. Rittmeyer A, Barlesi F, Waterkamp D, Park K, Ciardiello F, von Pawel J, Gadgeel SM, Hida T, Kowalski $\mathrm{DM}$, Dols MC, et al. Atezolizumab versus docetaxel in patients with previously treated non-small-cell lung cancer (OAK): a phase 3, open-label, multicentre randomised controlled trial. Lancet. 2017;389(10066):255-65.

50. Dong ZY, Zhang JT, Liu SY, Su J, Zhang C, Xie Z, Zhou Q, Tu HY, Xu CR, Yan LX, et al. EGFR mutation correlates with uninflamed phenotype and weak immunogenicity, causing impaired response to PD-1 blockade in non-small cell lung cancer. Oncoimmunology. 2017;6(11):e1356145.

51. Pascual J, Turner NC. Targeting the PI3-kinase pathway in triple-negative breast cancer. Annals of oncology: official journal of the European Society for Medical Oncology. 2019;30(7):1051-60.

\section{Figures}




\section{PTEN loss associated}
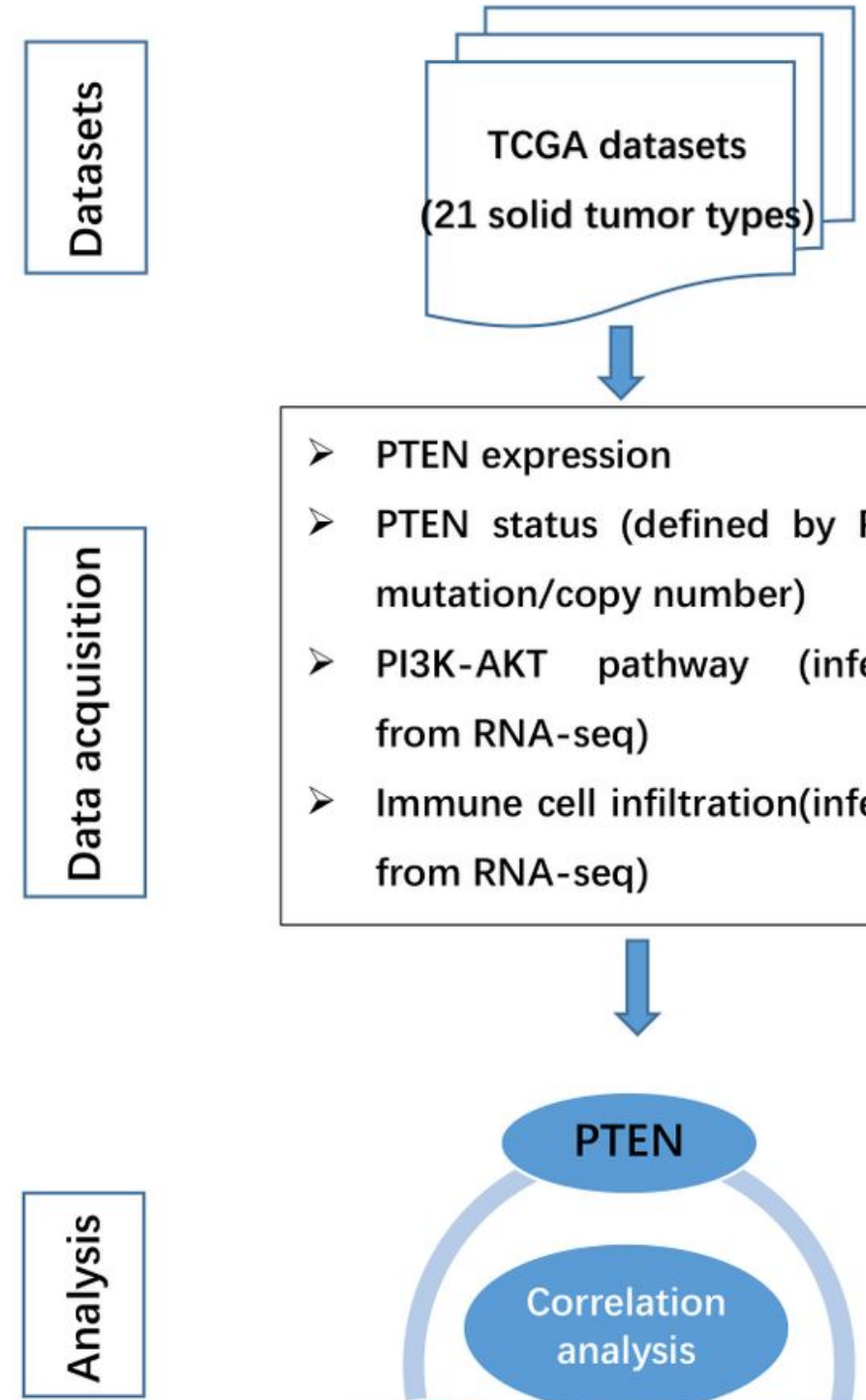

\section{PTEN}

\section{Correlation}

analysis with $\mathrm{T}$ cell exclusion

\section{PTEN expression predicted} response to immunotherapy
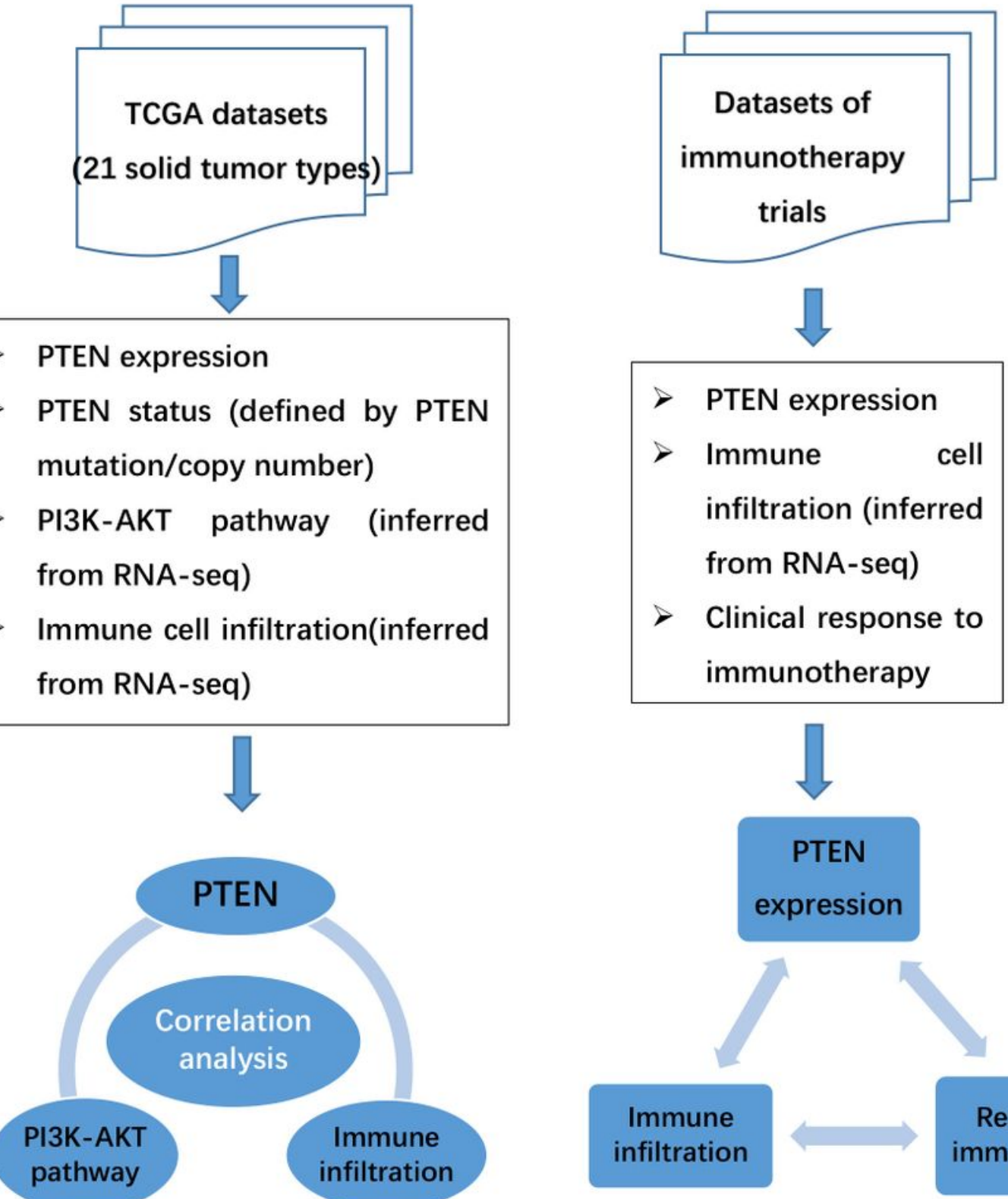

Immune

infiltration
Response to

immunotherapy

Figure 1

Flow scheme of the analysis process. 


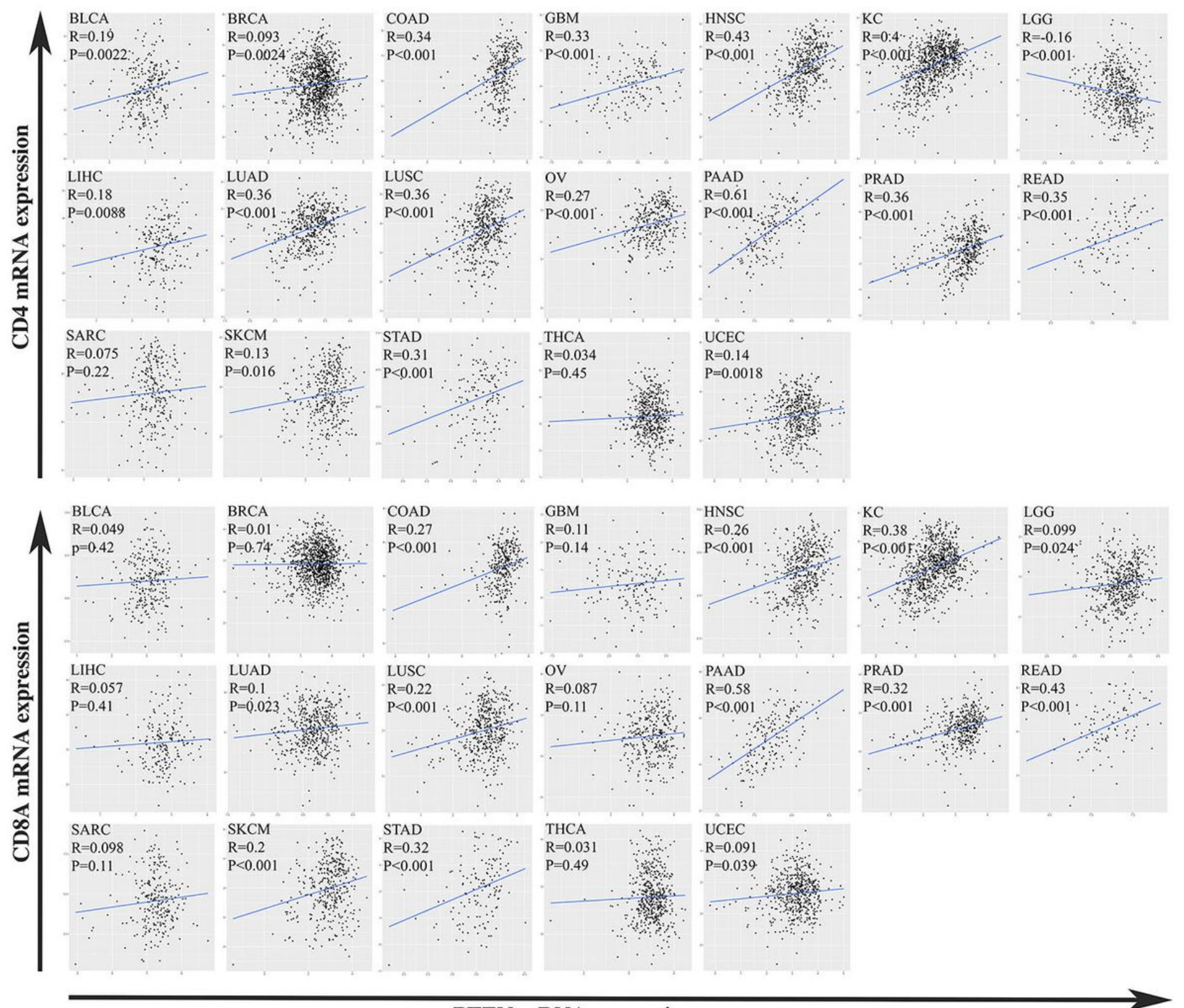

PTEN mRNA expression

\section{Figure 2}

Positive correlation between expression of PTEN and T-cell-inflamed genes Dot plots of PTEN mRNA expression (log transferred) on x-axis and T-cell-inflamed genes (CD4, CD8A) mRNA expression (log transferred) on y-axis per tumor types. correlation coefficients ( $R$ value) and $P$ value of Pearson Correlation were shown for each tumor type. mRNA expression of PTEN was positively correlated with expression level of CD4 and CD8 in multiple tumor types. Abbreviations: BLCA, Bladder Urothelial Carcinoma; BRCA, Breast invasive carcinoma; COAD, Colon adenocarcinoma; GBM, Glioblastoma multiforme; HNSC, Head and Neck squamous cell carcinoma; KICH, Kidney Chromophobe; KIRC, Kidney renal clear cell carcinoma; KIRP, Kidney renal papillary cell carcinoma; LGG, Brain Lower Grade Glioma; LIHC, Liver hepatocellular carcinoma; LUAD, Lung adenocarcinoma; LUSC, Lung squamous cell carcinoma; OV, Ovarian serous cystadenocarcinoma; PAAD, Pancreatic adenocarcinoma; PRAD, Prostate 
adenocarcinoma; READ, Rectum adenocarcinoma; SARC, Sarcoma; SKCM, Skin Cutaneous Melanoma; STAD, Stomach adenocarcinoma; THCA, Thyroid carcinoma; UCEC, Uterine Corpus Endometrial Carcinoma

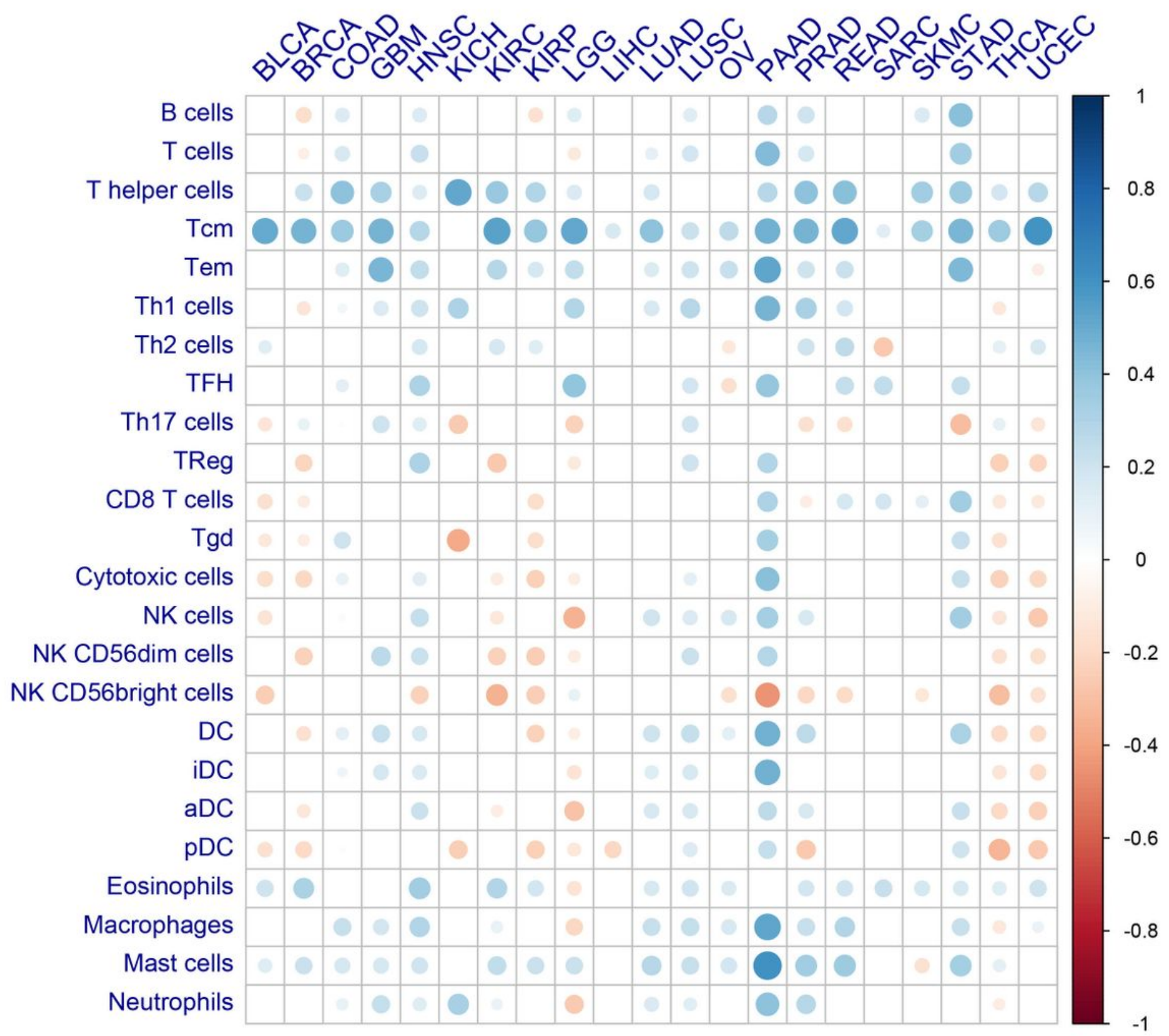

\section{Figure 3}

The Correlation between the Expression of PTEN and the Infiltration of Immune Cells The Correlation between the mRNA Expression of PTEN and 24 types of infiltrating immune cells was evaluated by Pearson Correlation analysis. Node color is determined by correlation, and node size indicates the significance of correlation. Only nodes with Correlation significance ( $P$ value) $<0.05$ were shown. PTEN expression was positively correlated with $T$ cells, $T$ helper cells, memory $T$ cells in multiple cancer types. Abbreviations: Th1, type $1 \mathrm{~T}$ helper cells; Th2, type $2 \mathrm{~T}$ helper cells; Tem, effector memory T cells; Tcm, central memory T cells; TFH, follicular T helper cells; TReg, regulatory T cells; Tgd, $\gamma \delta$ T cells; NK cells, 
nature killer cells; DC, dendritic cells; aDC, activated dendritic cell; pDC, plasmacytoid dendritic cell; iDC, immature dendritic cell; full terms of cancer abbreviation were shown in figure 2 .

PTEN白 Loss

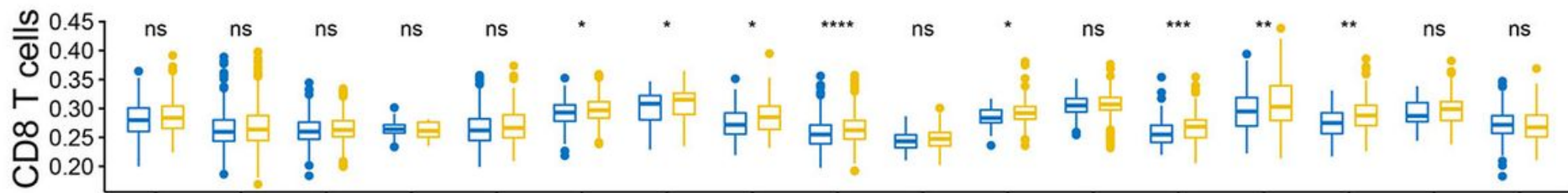

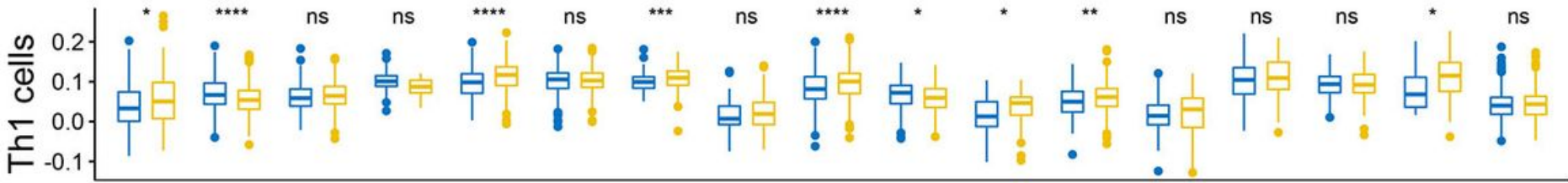
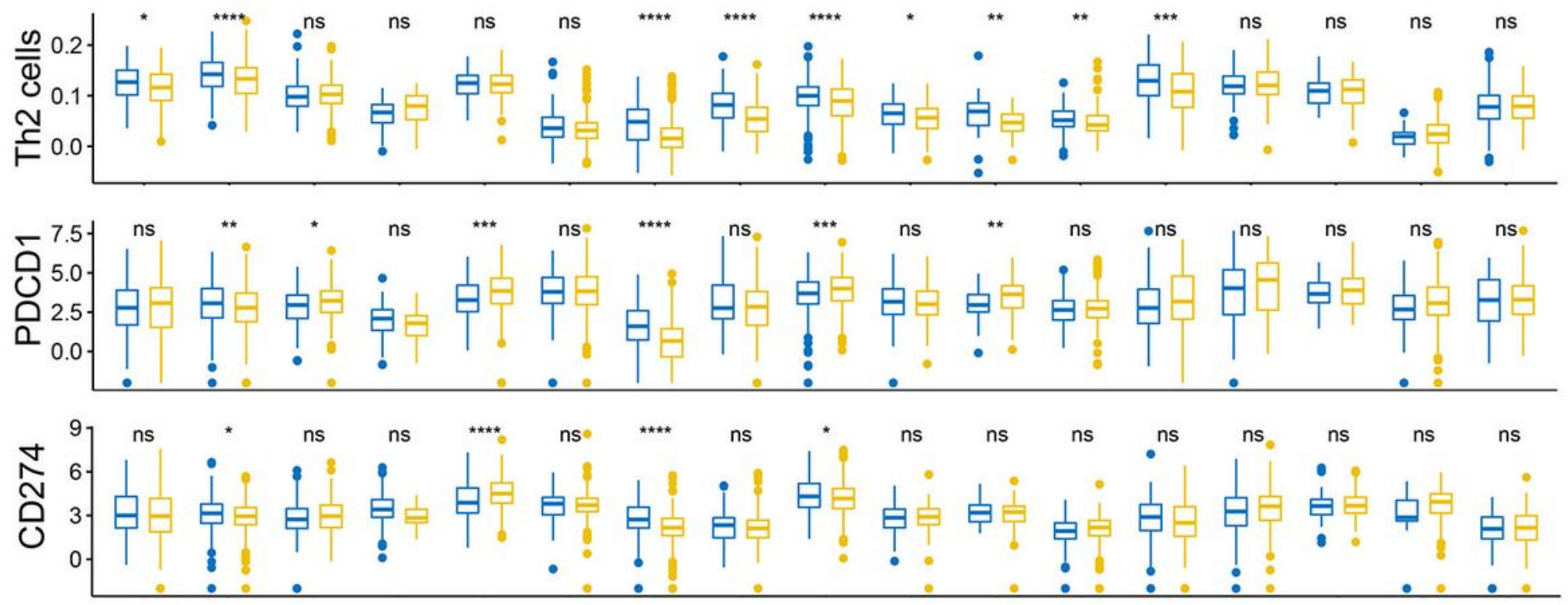

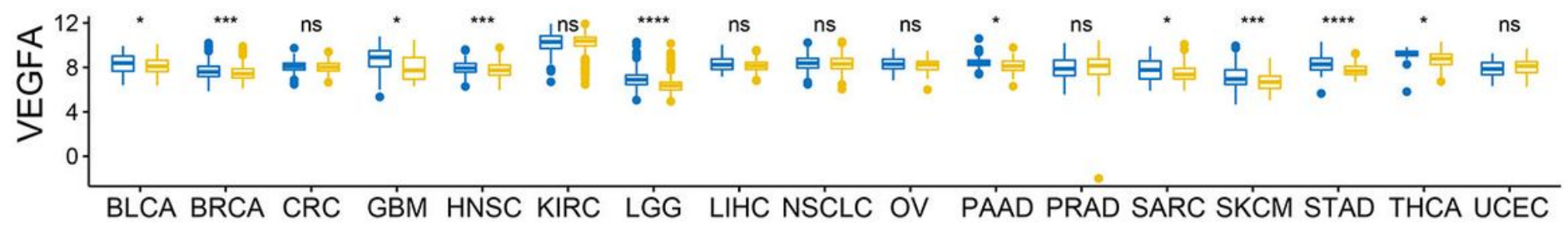

Figure 4

Difference of T cells infiltration or immunosuppressive markers between tumors with PTEN loss and tumors with Intact PTEN T cells (CD8+ T cells, Th1 cells, Th2 cells) infiltration level and mRNA Expression of Immunosuppressive markers (PD-L1, encoded by CD274; PD-1, encoded by PDCD1; VEGF, encoded by VEGFA) between tumor with PTEN loss or Intact PTEN. ${ }^{\star} p<0.05$; ${ }^{* \star} p<0.01$; ${ }^{\star \star \star} p<0.001$; ${ }^{* \star \star \star} p<0.0001$; ns, not significant. Tumors with PTEN loss demonstrated significant lower infiltrating level of CD8 T cell and type $1 \mathrm{~T}$ helper cells (Th1 cells) and expression level, but increased infiltration of type $2 \mathrm{~T}$ helper cells (Th2 cells) and expression of VEGFA in multiple cancer types. No general pattern was observed for the correlation of PTEN status with expression level of PDCD1 and CD274 among different cancer types. Abbreviations: full terms of cancer abbreviation were shown in figure 2 . 


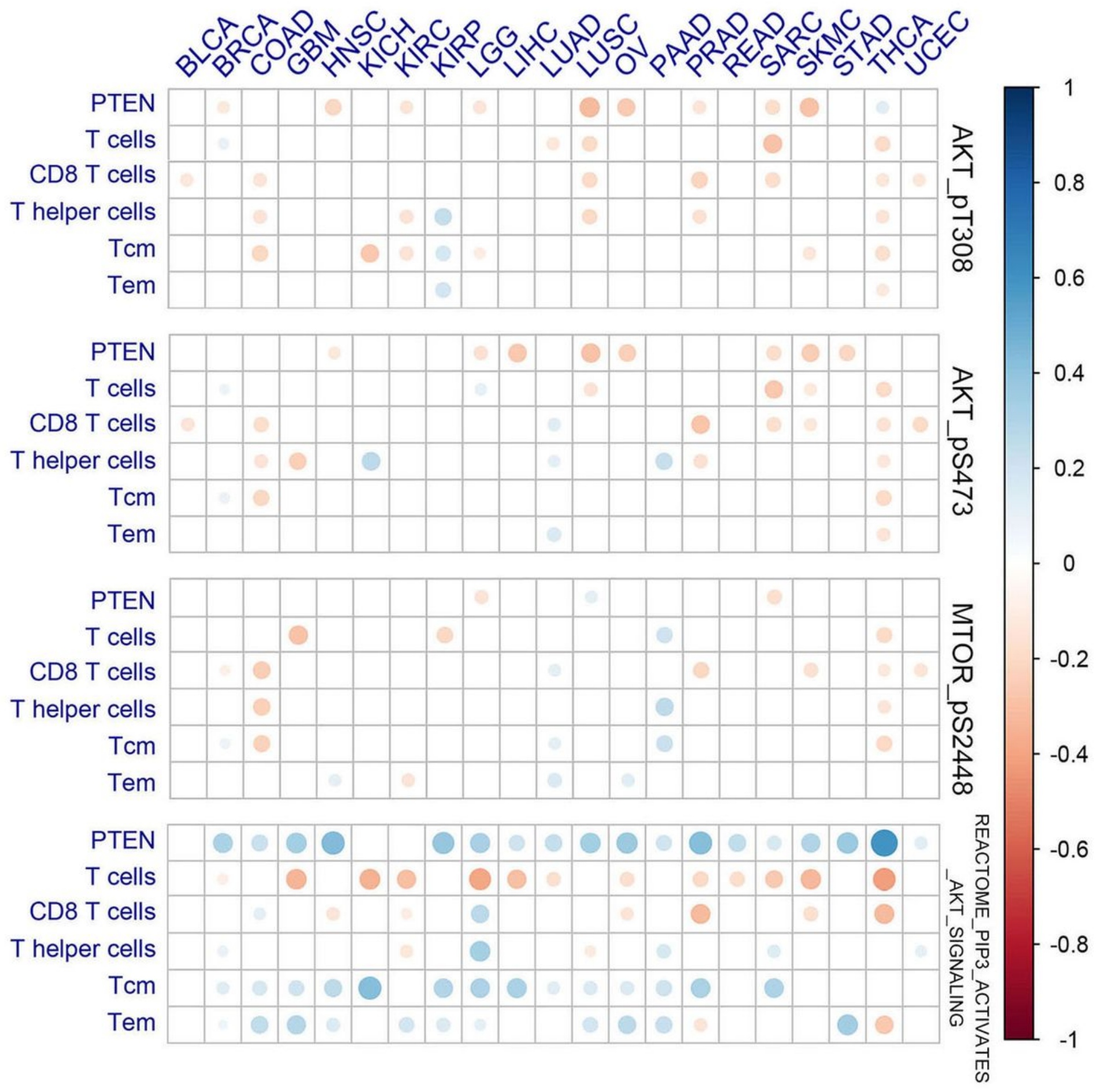

Figure 5

The Correlation of PI3K-AKT-mTOR pathway activation with PTEN expression and the T cells infiltration. The Correlation of PI3K-AKT-mTOR pathway activation (represented by p-AKT [phosphorylated at Thr308 or Ser473], p-mTOR[phosphorylated at Ser2448], and ssGSEA score of PI3K pathway) with PTEN expression and the T cells infiltration was evaluated by Pearson Correlation analysis. Node color is determined by correlation, and node size indicates the significance of correlation. Only nodes with 
Correlation significance (P value) $<0.05$ were shown. Abbreviations: Tem, effector memory T cells; Tcm, central memory T cells; full terms of cancer abbreviation were shown in figure 2.

a

$\begin{array}{ll}0.45 \\ \overline{0} & 0.40 \\ 0 & 0.35 \\ 0 & 0.30 \\ 0 & 0.25 \\ 0 & 0.20\end{array}$.

点 $\begin{array}{cc}0.1 \\ 0.1\end{array}$

BLCA BRCA CRC GBM HNSC KIRC KIRP LGG LIHC NSCLC OV PAAD PRAD SARC SKCM STAD UCEC

b

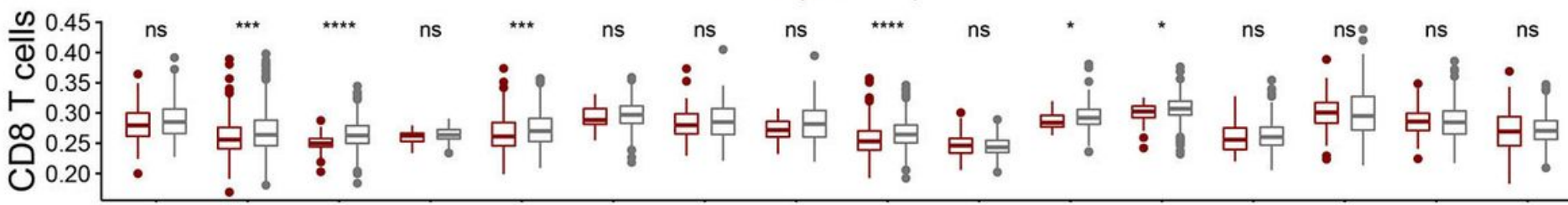

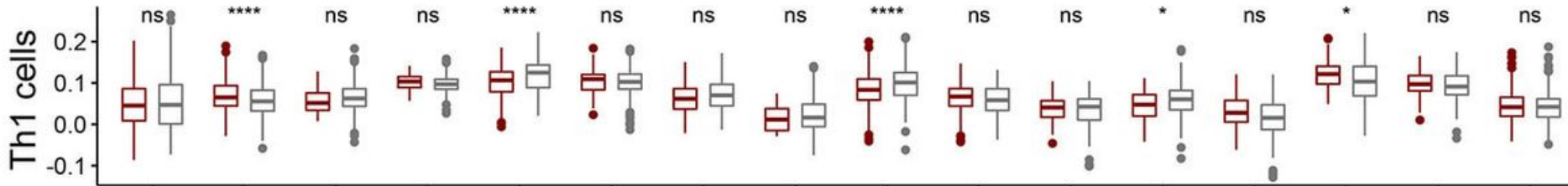

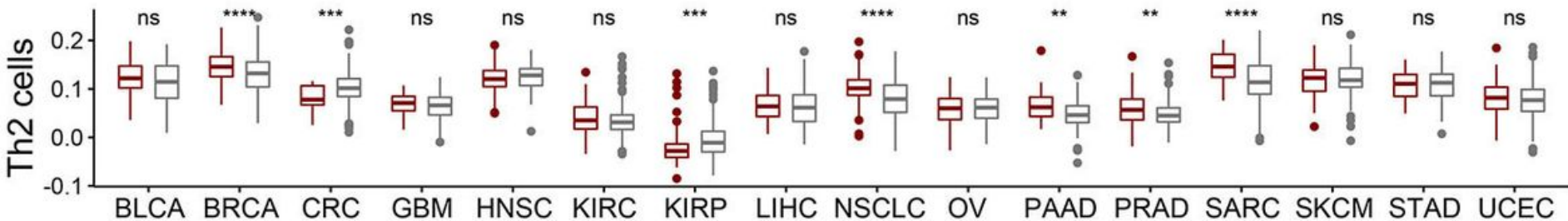

Figure 6

Difference of T cells infiltration between tumors with genomic gain in PIK3CA/PIK3CB and tumors with wide-type PIK3CA/PIK3CB (a) Difference of T cells infiltration between tumors with PIK3CA gain and tumors with wide-type PIK3CA; (b) Difference of T cells infiltration between tumors with PIK3CB gain and tumors with wide-type PIK3CB. ${ }^{*} p<0.05$; ${ }^{\star \star} \mathrm{p}<0.01$; ${ }^{\star \star \star} \mathrm{p}<0.001$; ${ }^{* \star \star \star} \mathrm{p}<0.0001$; ns, not significant. Genomic gain in PIK3CA/PIK3CB were associated with reduced infiltration of CD8 T cell and type $1 \mathrm{~T}$ 
helper cells (Th1 cells), but increased infiltration of type $2 \mathrm{~T}$ helper cells (Th2 cells) in multiple cancer types. Abbreviations: full terms of cancer abbreviation were shown in figure 2.
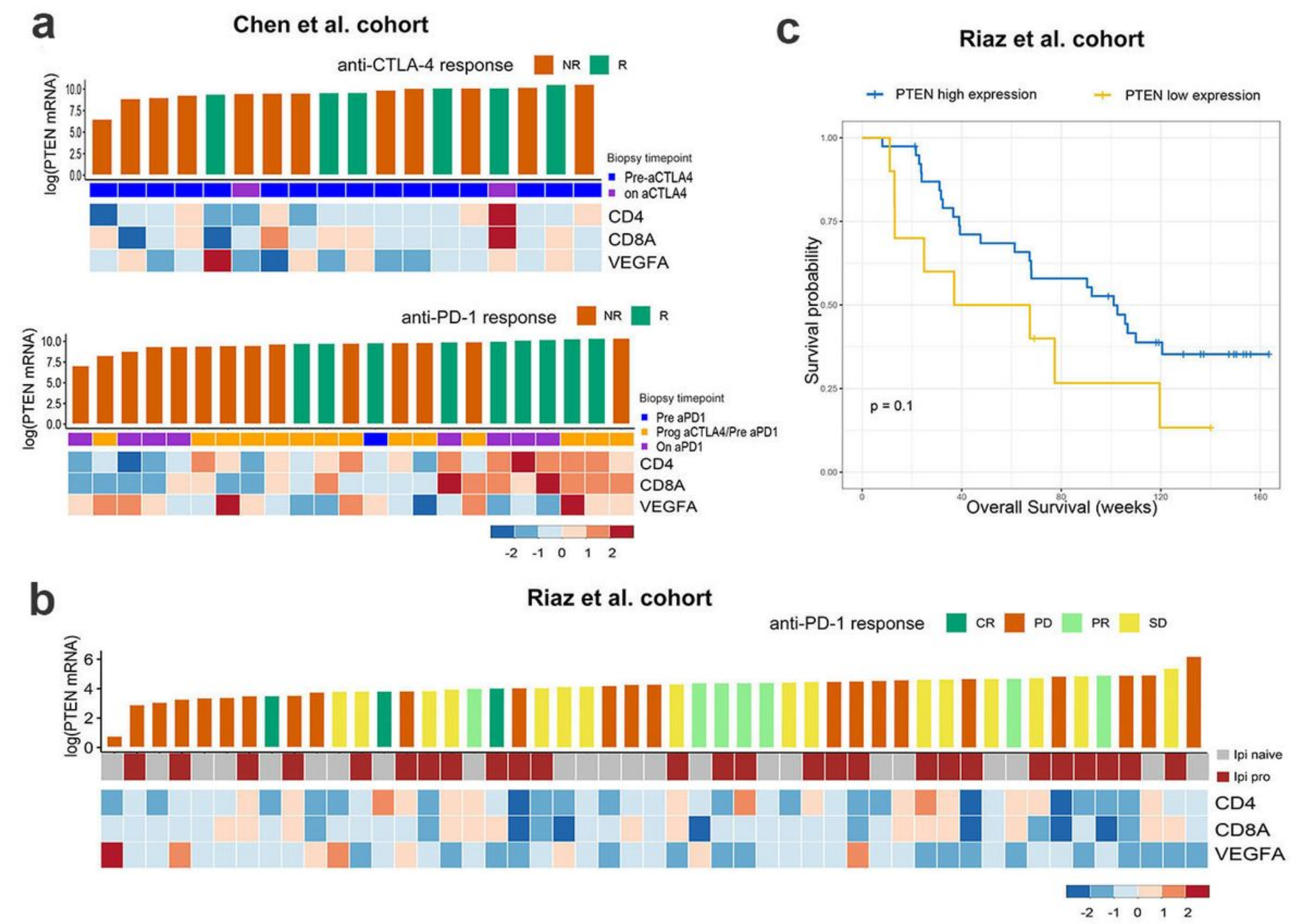

\section{Figure 7}

Predictive value of PTEN expression in patients receiving immunotherapy (a) Bar plot and heatmap demonstrated the mRNA expression (log transferred) level of PTEN and immune related genes (CD4, CD8A, VEGFA) respectively for patients receiving anti-CTLA4 blockade or anti-PD-1 blockade in Chen et al. cohort, with the samples ranked in the ascending order according to PTEN expression. Response to immunotherapy (R: response; NR: not response) was denoted by the color of the bar. Patients with higher PTEN expression demonstrated higher expression of T infiltration markers (CD4, CD8) and better response to CTLA-4 blockade or PD-1 blockade in Chen et al. cohort. (b) Bar plot and heatmap demonstrated the mRNA expression (log transferred) level of PTEN and immune related genes (CD4, CD8A, VEGFA) respectively for patients receiving anti-PD-1 treatment in Riaz et al. cohort, with the samples ranked in the ascending order according to PTEN expression. Response to immunotherapy (CR: complete response; PR: partial response; SD: stable disease; PD: progressed diseased) was denoted by the color of the bar. Patients with higher PTEN expression demonstrated higher expression of $T$ infiltration markers (CD4, CD8) and better response PD-1 blockade in Riaz et al. cohort. (c) Kaplan-Meier 
plots of overall survival difference between patients with low PTEN expression (the bottom fifth, $n=10$ ) and high PTEN expression (the top four fifths, $n=39$ ) in Riaz et al. cohort. Patients with higher PTEN expression demonstrated improved overall survival after anti-PD-1 immunotherapy in Riaz et al. cohort.

\section{Supplementary Files}

This is a list of supplementary files associated with this preprint. Click to download.

- Supplementaryfigurelegends.docx

- sFig1.jpg

- sFig2.jpg

- sFig3.jpg

- sFig4.jpg

- sfig5.jpg

- supplementarytables.xIsx 